\title{
Clinical and molecular aspects of the pathogenesis of Staphylococcus aureus bone and joint infections
}

\author{
R. CUNNINGHAM, A. COCKAYNE and H. HUMPHREYS \\ Public Health Laboratory and Division of Microbiology, Department of Clinical Laboratory Sciences, University \\ Hospital, Queen's Medical Centre, Nottingham NG7 2UH
}

\begin{abstract}
Staphylococcus aureus is an important cause of bone and joint infections. In recent years, significant changes in the incidence of septic arthritis and osteomyelitis have occurred. Haematogenous osteomyelitis is now less common during childhood, but secondary spread of infection to bone or joint from a contiguous site in adults is increasing in incidence. Infection introduced at the time of surgery or arising by the haematogenous route is a significant complication of prosthetic joint implantation, and the effect of bone cement on local immune function may be important in this setting. Although $S$. epidermidis is a more common cause of prosthetic joint infection, $S$. aureus is more difficult to treat. $S$. aureus produces a number of extracellular and cellassociated factors, but it is unclear what role these have as virulence factors in vivo. Furthermore, it is difficult in animal models to simulate transient bacteraemia followed by non-fulminating septic arthritis or osteomyelitis, as occurs in the patient. Surface factors which may be important in pathogenesis include the cell wall (activates complement and stimulates cytokine release), capsular polysaccharide (promotes adhesion to host cell surfaces), collagen receptors and fibronectin-binding protein. Staphylococcal toxic shock syndrome toxin (TSST-1) and the enterotoxins are superantigens and have the potential to suppress plasma cell differentiation and antibody responsiveness. TSST-1-positive isolates have been shown to cause more severe joint infection in one animal model, but most other studies to date have focused on in-vitro rather than in-vivo effects. There is little evidence supporting a role for coagulase, lipase and the haemolysins in staphylococcal bone and joint infections. Despite the clinical importance of these infections, surprisingly little is known about pathogenesis at the cellular level. Future research should focus on the role of the host immune system in limiting spread of infection, and the expression of virulence factors in animal or other models incorporating isogenic mutant strains.
\end{abstract}

\section{Introduction}

Staphylococcus aureus is a well-recognised cause of bone and joint infections. Infections may occur as a complication of septicaemia - i.e., haematogenous infection - or following local trauma. Pathogenesis is multi-factorial and poorly understood at present. Host factors such as underlying disease, immune status and virulence determinants of the organism are all likely to be important. New molecular techniques to determine the relative importance of the many potential virulence factors, and studies into the interaction between these

Received 5 July 1995; accepted 4 Aug. 1995. and the host's immune response (e.g., cytokine production), are likely to provide a greater insight into pathogenic mechanisms. The purpose of this article is to review the evidence for the role of the potential virulence factors in $S$. aureus bone and joint infection, and to suggest suitable areas for future research.

\section{Clinical aspects}

\section{Classification}

The conventional classification of bone and joint infections incorporates aspects of pathogenesis (i.e., haematogenous or non-haematogenous) and duration of infection (i.e., acute or chronic). Haematogenous 
osteomyelitis is more common during childhood and is characterised by an acute febrile illness with pain and immobility of the affected limb. Blood cultures are positive in $c .50 \%$ of cases [1]. In contrast, bacteraemia caused by $S$. aureus in adults results in osteomyelitis rarely, and secondary spread of a contiguous focus of infection, such as from a surgical wound, is more common. Infective or septic arthritis usually follows haematogenous inoculation, and $S$. aureus is the most common organism isolated. Risk factors include rheumatoid arthritis, osteoarthrosis and intra-articular injections [2]. Mader and colleagues [3,4] have devised a novel classification of osteomyelitis that takes account of the anatomical site, the immunocompetence of the host and other factors. This approach has the advantage that certain factors may be linked to prognosis and specific treatment protocols devised accordingly, but provides little insight into pathogenesis. Haematogenous osteomyelitis is more common in infants and children because of the anatomy of blood flow in the long bones. Arterial blood flow leads to non-anastomotic capillaries which enter large venous sinusoids where flow may become slow and turbulent. Injury to such a site may result in haematoma and necrosis, thereby increasing the susceptibility to infection during a transient bacteraemia [3].

\section{Epidemiology}

Several recent reviews have confirmed the importance of $S$. aureus as a cause of both contiguous and haematogenous osteomyelitis [5-7], although Haemophilus influenzae type B rivalled $S$. aureus as a cause of acute septic arthritis until the introduction of the conjugated vaccine [6]. A history of trauma or skin infection is a significant risk factor for bone and joint infections caused by $S$. aureus $[5,6]$, presumably because of the propensity of this bacterium to colonise broken skin.

Although septic arthritis and osteomyelitis remain important clinical conditions, changes in incidence are occurring, especially in children. Two recent studies have documented a decline in haematogenous osteomyelitis accompanied by a rise in contiguous disease [8,9]. In a review of over 15000 cases of $S$. aureus bacteraemia with 700 cases of osteomyelitis in Denmark, Esperson and colleagues [8] recorded a decrease in bone and joint infections from $7 \cdot 1 \%$ of bacteraemic patients between 1959 and 1963 to $3.3 \%$ between 1969 and 1973. This occurred at a time when the overall incidence of $S$. aureus bacteraemia was increasing. Haematogenous osteomyelitis was usually community-acquired, whereas contiguous infection was much more likely to be acquired in hospital. While the number of patients aged between 1 and 20 years fell, those aged over 50 years increased to comprise $55 \%$ of the total [8]. A 20-year survey of hospital records of children admitted to hospital in Glasgow with osteomyelitis revealed that $S$. aureus was the most common aetiological agent, but the proportion of children with less clinically florid infection increased from $12 \%$ to $42 \%$ over the period studied [9]. These changes may be related to improved living standards and earlier diagnosis. The initiation of broad-spectrum antibiotics before a definitive diagnosis is made may explain the rise in sub-acute cases in which infection is suppressed but not eradicated.

With the fall in incidence in the paediatric age group, there is increasing interest in adult bone and joint infections, especially in the elderly. In a review of 23 patients with septic arthritis, 15 had pre-existing joint disease and eight patients had one or more chronic systemic illnesses such as diabetes mellitus, neoplasia or liver cirrhosis [10]. Mortality in this group of patients was $>20 \%$, but this relatively high figure may have been related to the severity of underlying disease. Adult osteomyelitis may occur at any site, but infection of the vertebral column is becoming relatively more common [8]. Most of these cases occur in older patients; 108 of 140 patients were aged over 50 years in one recent study [8].

With the increased prevalence of methicillin-resistant $S$. aureus (MRSA) in many hospitals, infections caused by antibiotic-resistant strains are becoming more common. Osteomyelitis caused by MRSA often follows multiple trauma, and is usually hospitalacquired. All 10 patients described by Fitzpatrick and colleagues [11] were admitted to hospital after road traffic accidents, and in nine patients the infection was localised to the site of trauma. Osteomyelitis caused by MRSA is not confined to adults and has also been reported in neonatal units. Between 1981 and 1987, 200 episodes of bacteraemia occurred in an Australian neonatal unit, of which 14\% were caused by MRSA [12]. Twenty cases of osteomyelitis or septic arthritis were identified - of which $75 \%$ were in infants of low birth weight - and three patients died [12]. In contrast to the disease in adults, where MRSA bone and joint infection is usually localised, all the neonates had signs and symptoms of systemic infection, such as temperature instability, poor perfusion and lethargy [12].

\section{Prosthetic joint infection}

Prosthetic joint implantation has now become a common surgical procedure, and the marked improvement in joint function that follows represents one of the great medical advances of recent years. However, joint infection is a significant complication and is associated with protracted hospitalisation, disability and considerable additional medical expense [13]. Infection may occur by the haematogenous route or be introduced at the time of surgery, and although $S$. epidermidis and other coagulase-negative staphylococci are more common pathogens in this setting, infection caused by $S$. aureus is often difficult to treat with antimicrobial 
agents alone, and removal of the prosthetic joint is usually necessary [13]. Risk factors for prosthesis infection include rheumatoid arthritis, skin necrosis and post-operative wound infection [13-15]. The effect of bone cement, which is often required to ensure firm implantation, on local immune function has been the subject of much interest. Methylmethacrylate monomer is released in small concentrations during and for a time after polymerisation, and this has been shown in experiments in vitro to partially abolish the inhibitory effect of serum on staphylococci [16]. Other studies have demonstrated increased survival of bacteria in the presence of methylmethacrylate, probably because of inhibition of phagocytosis [17]. These studies are limited by a lack of information on the concentration of cement present at the bone-joint interface.

\section{Pathogenesis}

\section{S. aureus virulence factors and their analysis}

'In-vitro studies reproduce but imperfectly the conditions present in the intact host, and in our present state of ignorance only the crudest chemical changes can be followed.' S. D. Elek [18].

Staphylococcal virulence factors are those products that enable the organism to establish infection and enhance its potential to cause disease. $S$. aureus produces a large number of extracellular and cellassociated factors that may contribute to virulence. With the exception of a few well-characterised toxins - e.g., TSST-1 and the enterotoxins - the relevance of many putative virulence factors in disease causation has been difficult to establish. Before the development of methods for the creation of isogenic mutants, it was necessary to purify individual bacterial components and test their effect in an in-vitro assay or in an appropriate animal model. Difficulties in the purification of minor, often labile, staphylococcal products in an active form limited the success of this approach. Similarly, the development of suitable in-vitro assays and animal models that accurately reflected the situation during infection in vivo made progress difficult. Although testing the effect of individual purified components in animal models may yield some useful information, it provides no insight into the potential synergic interactions between different factors which may occur in vivo. This type of approach also fails to take into account the dynamic nature of the interactions between the bacterium and the host during infection, and the variable role of different bacterial products at different stages in disease progression.

The increasing recognition that bacterial phenotype including the expression of putative virulence determinants - is influenced by growth conditions also has major implications for identification of staphylococcal virulence factors. For logistic reasons, most studies have utilised bacteria grown in vitro in nutrient-rich broth culture [19]. In contrast, conditions in vivo are usually nutrient-limited, particularly for key elements such as iron [20], and bacteria grow most commonly in association with a solid substrate rather than in the planktonic mode [21]. In addition, since many staphylococcal products are only expressed at certain times in the growth cycle, the growth conditions used to prepare the bacteria may significantly affect the phenotype expressed and the behaviour of the organism in a given assay [20].

Despite these potential problems, the increasing use of molecular methods to create isogenic mutants and the continuing development and refinement of in-vitro and animal models has allowed some progress to be made in analysing the role of several staphylococcal products in disease. Studies performed specifically in relation to bone and joint infections are relatively few, but the possible role of several of these bacterial products in the pathogenesis of these infections are discussed in the following sections and summarised in Table 1 [22-37].

\section{Animal models of $S$. aureus bone and joint infections}

Animal models that reflect the clinical and pathological changes associated with $S$. aureus bone and joint infections accurately are essential in elucidating the mechanisms of pathogenesis. Several animal species

Table 1. Putative virulence factors in $S$. aureus bone and joint infections

\begin{tabular}{lll}
\hline Virulence factor & \multicolumn{1}{c}{ Action } & Role* [references] \\
\hline $\begin{array}{l}\text { Cell-associated } \\
\text { Collagen-binding protein }\end{array}$ & Adheres to type 1 collagen & Probable [22-24] \\
$\begin{array}{l}\text { Fibronectin-binding protein } \\
\text { Protein A }\end{array}$ & Adheres to fibronectin \\
Extracellular & Binds immunoglobulins & Probable [25, 26] \\
Enterotoxins & & Possible [25, 27] \\
TSST-1 & Immunomodulatory & Possible [28-30] \\
Others & Immunomodulatory & Probable [31-33] \\
Capsular polysaccharide & & Possible [34] \\
Coagulase & Protective & Doubtful [35] \\
Haemolysins & Protective & Possible [35, 36] \\
Lipase & Immunomodulatory & Doubtful [37]
\end{tabular}

*In $S$. aureus bone and joint infections. 
have been used to model acute and chronic posttraumatic osteomyelitis [38], where bacteria are implanted directly into bone, usually in the presence of sclerosing agents and foreign bodies. More recently, Spagnolo et al. [39] modified this method by using fibrin glue in place of other agents to initiate infection with $S$. aureus, and produced many of the clinical and pathological changes found in chronic osteomyelitis. Models for acute osteomyelitis acquired by the haematogenous route have also been developed [40]. A rat model of $S$. aureus arthritis following administration by the i.v. route has also been described [41]. Induction of arthritis was characterised by infiltration of the synovium with $\mathrm{T}$ lymphocytes and phagocytes, an increase in the serum level of interleukin 6 , and by polyclonal B cell activation. This study highlights one of the major limitations of animal studies in that the i.v. injection of $S$. aureus results in death, or a very acute fulminating infection, in a high proportion of animals. This contrasts with the situation in the patient where, following a transient bacteraemia, septic arthritis or osteomyelitis has a much less florid presentation.

\section{Virulence factors}

\section{Surface factors}

In common with other bacterial pathogens, there has been an increased recognition of the importance of staphylococcal surface components as virulence determinants. As the bacterial surface is the site of interaction with host tissues and immune effectors, it plays a key role in the disease process. The cell wall consists of a peptidoglycan layer, with associated teichoic acids, lipoteichoic acids and proteins. Both peptidoglycans and teichoic acids can activate the alternative complement pathway, induce platelet aggregation in the presence of protein $\mathrm{A}$, and stimulate interleukin 1 release from human monocytes [42]. These are all considered significant in the development of septic shock, but what impact these actions have in bone and joint infection is unknown. The majority of $S$. aureus isolates are also capsulate. These structures are, potentially, involved in a number of processes relevant to infection. These include adhesion to host cell surfaces, modulation of interactions with immune effectors, including antibody and phagocytes, and stimulation of cytokine release from $\mathrm{T}$ lymphocytes. Capsulate strains have also been shown to stimulate cytokine release from epithelial and endothelial cells and from monocytes, an effect which is inhibited by serum [43]. Foster and McDevitt [25] recently reviewed the possible roles of surface-associated proteins including protein $\mathrm{A}$, fibronectin-binding proteins, fibrinogenbinding protein and collagen-binding protein, and particularly highlighted the importance of fibronectinbinding proteins in adherence to biomaterials coated with host proteins in vivo, a factor of potential importance in prosthetic joint infection. In addition, strains of $S$. aureus isolated from osteomyelitis and septic arthritis usually adhere to collagen and cartilage in vitro - a property which is mediated by a surfaceassociated adhesin - and it was speculated that bone infections might be prevented by a vaccine derived from this [25].

Adherence. The propensity of $S$. aureus to adhere to cartilage and bone is undoubtedly important in pathogenesis, thus explaining the high incidence of bone and joint infection following bacteraemia. Williams and colleagues [44] studied the RNA and cellassociated protein synthesis in an in-vitro model of adult chondrocytes following inoculation with $S$. aureus. Overall protein synthesis was inhibited by $84 \%$ in cells exposed to $S$. aureus, with an increased release of collagenase and gelatinase. It was suggested that potential proteolytic activity present in normal joints remains inhibited in the absence of infection [44]. In a mouse model, there was significant binding of $S$. aureus to bone sialoprotein, fibronectin and collagen type 1 , indicating that adherence remains a key phase in the early stages of infection [45]. A recent study investigated the agglutination of $S$. aureus isolates from patients with osteomyelitis to collagencoated latex beads. In an ${ }^{125} \mathrm{I}$-collagen-binding assay, the binding activity was 20 -fold lower at $42^{\circ} \mathrm{C}$ compared with $37^{\circ} \mathrm{C}$. This alteration in binding activity was associated with an altered polypeptide profile on SDS-PAGE [22].

Collagen-binding proteins. There is convincing evidence that a collagen-binding protein is important in the pathogenesis of osteomyelitis and septic arthritis. A cell-surface protein that mediates attachment of $S$. aureus to cartilage has been cloned and sequenced [23]. In previous experiments it was also shown that virtually all strains isolated from patients with osteomyelitis or septic arthritis possessed a collagen receptor, whereas this is only expressed in one-third of isolates from soft tissue infection [24]. Antibodies to the receptor blocked staphylococcal binding to cartilage, as did pre-incubation with a recombinant form of the receptor protein. Inoculation of mice i.v. with mutants positive and negative for the collagen adhesin gene showed that septic arthritis occurred with $>70 \%$ of the positive strains, but with only $27 \%$ of the negative strains. The strains positive for collagen adhesin were also associated with the production of higher levels of IgG and interleukin-6 [24].

Fibronectin-binding proteins. Fibronectin is a protein that, along with fibrinogen, rapidly coats any foreign body implanted in a patient. It occurs in a matrixassociated form which is exposed after damage to endothelial cells and is also found in blood clots. Two fibronectin-binding proteins with significant homology have been described. It has been demonstrated that, while intravenous cannulae are coated with higher concentrations of fibrinogen than fibronectin, the fibronectin is more important in binding of $S$. aureus 
because fibrinogen is degraded by plasmin to such a degree that bacterial adhesion is impaired [26]. An invivo study with a rat model of endocarditis showed that mutants deficient for fibronectin-binding protein were 250-fold less adherent to traumatised heart valves, although no studies have yet been reported for bone and joint infections [46]. It is likely that fibronectinbinding proteins play a role in bone and joint infections - especially those associated with prosthetic joints and when haematoma, a source of fibronectin, are present.

Capsular polysaccharide. Most (>90\%) S. aureus strains produce capsular polysaccharide. It has been detected in strains from both invasive infection and asymptomatic carriage. However, in a rat endocarditis model, the most common capsular serotype (serotype 8 , found in $53 \%$ of isolates) did not demonstrate any increase in virulence over non-capsulate mutant strains [34]. Strains producing capsules of serotypes 1 and 2 are highly virulent in animal models, but have only been reported rarely as clinical isolates [47]. $S$. aureus capsular polysaccharide serotype 8 (CP8) is expressed maximally by organisms cultured on a solid surface. Iron-restricted conditions also induced expression of CP8 [48]. It remains to be determined whether capsules play any role in staphylococcal bone and joint infection.

Protein $A$. This is a $42-\mathrm{kDa}$ protein bound covalently to the outer peptidoglycan layer of the $S$. aureus cell wall. It is also found in the supernate from broth cultures, although the concentration varies according to the strain and detection technique used. Expression of protein A by $S$. aureus is regulated by the accessory gene regulator $(a g r)$. This is a global regulator that alters the expression of many genes in $S$. aureus, including those coding for haemolysins and enterotoxins. Protein A has been postulated as a virulence factor in $S$. aureus infection as it binds to the Fc portion of all IgG subclasses except $\operatorname{IgG}_{3}$. Protein $A$ can also bind to Fc receptors on polymorphonuclear leukocytes interfering with opsonisation and phagocytosis. This has been demonstrated by in-vitro phagocytosis studies with a strain that produces protein $\mathrm{A}$ and a negative mutant [27]. An animal model incorporating subcutaneous abscesses and peritonitis demonstrated that a strain producing protein $\mathrm{A}$ was more virulent than a negative mutant. This work has not been repeated to date in animal models of bone and joint infection [49].

\section{Secreted proteins}

Enterotoxins. The staphylococcal enterotoxins are a group of seven proteins (SEA, SEB, SEC1, SEC2, SEC3, SED and SEE), all of which have a mol. wt of $26-30 \mathrm{kDa}$. There is significant DNA sequence homology between the toxins (75-99\%), with the exception of SED which has $55 \%$ homology. These toxins induce vomiting and diarrhoea when ingested, but also have been shown to exert a profound effect on the immune system when administered parenterally. They act as superantigens and suppress plasma cell differentiation in vitro [28] and antibody response in vivo [29]. Enterotoxins also induce production of cytokines, such as interferon-gamma, tumour necrosis factor, interleukin 2 and interleukin 6 by $T$ cells and monocytes. These act synergically, and the relative contribution of each cytokine to the pathogenesis of staphylococcal bone and joint infection is unclear. Sourek et al. [30] studied 264 strains of $S$. aureus from patients with chronic osteomyelitis and found that 79 produced one or more enterotoxin. It was concluded that because only $30 \%$ of the isolates were positive, enterotoxin production alone was not a major factor in pathogenesis.

Toxic shock syndrome toxin (TSST-1). This exotoxin came to prominence because of a well-defined clinical syndrome associated with the use of high-absorbency tampons. The syndrome comprises fever, shock and a desquamating rash, often with organ failure, but most cases are not associated with bacteraemia. A 22-kDa protein (TSST-1) has been detected in virtually all isolates of $S$. aureus from affected patients. TSST- 1 has very little sequence homology with the enterotoxins, despite occasional reports of similar clinical presentations where strains producing only enterotoxins have been isolated. It is a known superantigen, stimulating non-specific T-cell proliferation by interacting directly with class II major histocompatibility complex molecules, leading to massive lymphokine and monokine release [50]. It has been shown that expression of TSST-1 is increased when organisms are cultured in media deficient in magnesium [51]. Binding of magnesium by fibres used in the production of tampons is thought to lead to high levels of TSST-1 in vivo. Many cases have been associated with bacterial growth in surgical dressings, but we are unaware of any cases associated with staphylococcal bone and joint infections. This is not surprising since many strains producing TSST-1 are not pyogenic, possibly because of massive TNF- $\alpha$ release from macrophages [31]. TSST-1 production is also maximal at neutral $\mathrm{pH}$, a condition which is unlikely to apply in an osteomyelitic abscess. TSST-1 may be of greater importance in septic arthritis. Animal studies of mice given a strain of $S$. aureus producing TSST-1 by i.v. injection demonstrate that a septic polyarthritis is developed readily. For the first $48 \mathrm{~h}$, a predominantly polymorphonuclear leukocyte and macrophage inflammatory response is seen but, after $72 \mathrm{~h}$, the proportion of CD4-positive T cells increases to $20 \%$. These carry distinct VB receptors, suggesting clonal expansion in the septic joint [32]. When the model was extended by infecting mice with strains of $S$. aureus isogenic for TSST-1, the mice infected with strains secreting TSST-1 developed more frequent and severe arthritis, and greater interleukin-2 receptor expression in synovial cells was demonstrated 
[33]. It is not yet clear to what extent this results from the superantigenic properties of TSST- 1 .

\section{Other potential virulence factors}

Coagulase. This enzyme is produced by virtually all strains of $S$. aureus. It exists in both free and bound forms, and stimulates plasma clotting by complexing with prothrombin. Bound coagulase may also activate complement. Its detection has been used as the primary identifying character of $S$. aureus. The expression of coagulase appears to be regulated negatively by agr. Phonimdaeng et al. [52] demonstrated that coagulase levels were higher in a strain with a mutation in the $a g r$ locus. Since coagulase-negative staphylococci are generally considered to be less virulent than $S$. aureus, it has been assumed that coagulase is a virulence factor. Coagulase-deficient mutants of $S$. aureus have been shown to be less virulent in a mouse mastitis model [35]. The thrombin produced by coagulase is lysed in vitro by proteolytic enzymes in normal serum, so it is unlikely that it protects infecting organisms for long periods of time [18]. However, it is possible that a thin layer of fibrin may inhibit phagocytosis for long enough to allow organisms to become established on bone or synovium, following which other protective mechanisms may come into play.

Haemolysins. $\alpha$-Haemolysin is an exotoxin produced by $90 \%$ of $S$. aureus strains. It causes lysis of erythrocytes of many species, including those of the horse and rabbit, although human erythrocytes are relatively resistant. $\alpha$-Haemolysin monomers are hydrophilic and have a molecular size of c. $34 \mathrm{kDa}$. They can bind to the cell membranes of many cell types, including erythrocytes, platelets, monocytes and endothelial cells [36]. Following binding, the monomers aggregate to form a ring-shaped hexamer with a hydrophilic channel in the centre. This allows the rapid diffusion of low mol. wt molecules and ions, such as calcium, and causes lysis or death of the cell within minutes. The expression of toxin is also regulated by $a g r$, expression being increased by an intact agr locus. $\alpha$-Toxin has been shown to be a virulence factor in animal models of peritonitis and mastitis, although its role in bone and joint infection has not been confirmed [35]. $\beta$-Haemolysin is a phospholipase which can be demonstrated in vitro by incubating blood agar plates at $4^{\circ} \mathrm{C}$ after incubation at $37^{\circ} \mathrm{C}$. There is no evidence that it plays a role in bone and joint infection. In the past, antibodies to $\gamma$-haemolysin have been assessed as a method of diagnosis of bone and joint infection, but it is unclear what significance should be attached to this in terms of pathogenesis [53].

Lipase. Virtually all strains of $S$. aureus are lipolytic, although there is believed to be more than one isoenzyme and these are not substrate specific [54]. The lipases produced by other organisms, such as Pseudomonas aeruginosa and clostridia, are not thought to cross-react with those produced by staphylococci. An ELISA used to detect IgG antibodies to lipase was found to be $88 \%$ sensitive and highly specific when used to differentiate $S$. aureus endocarditis from uncomplicated septicaemia. However, when the same assay was applied to patients with chronic osteomyelitis, only two of 26 patients were positive, with another four patients showing a significant rise in titre during the active stage of their infections [37]. Whether this was caused by reduced expression of lipase under the conditions found in chronic osteomyelitis, or a deficient serological response in this patient group, is unclear. In summary, staphylococcal lipase has never been proven to be important in the pathogenesis of any staphylococcal infection.

\section{Conclusions}

The epidemiology of $S$. aureus bone and joint infection has changed in recent years, with a relative fall in paediatric cases, an increasing incidence of prosthetic joint infection, and MRSA posing a major challenge in treatment and control. There have also been favourable changes in the way investigations of pathogenicity are conducted, with less reliance on in-vitro characteristics, more sophisticated animal models and molecular techniques allowing the creation of isogenic mutants positive and negative for individual virulence factors.

The pathogenesis of haematogenous bone and joint infection and the role of staphylococcal virulence factors is undoubtedly complex. It may be argued that as organisms with low pathogenic potential - e.g., $S$. epidermidis - can thrive when inoculated into bone tissue or a joint, virulence factors are of little relevance. This may be so when organisms are inoculated directly into the joint space, a rare event in practice, but the likely mechanism of haematogenous infection - i.e., the adherence of staphylococci to capillary endothelium following minor trauma suggests an obvious role for virulence factors [55]. There is good evidence that expression of collagenbinding proteins increases the likelihood of staphylococcal adherence. Other factors may assist in avoidance of opsonisation and phagocytosis following adherence. These may include capsular polysaccharide and protein A. Finally, more sophisticated factors - e.g., the enterotoxins and TSST-1 - may act to subvert the cellular and humoral immune system. These may be the crucial factors which determine whether a microscopic abscess is eliminated or develops into full-blown osteomyelitis or septic arthritis. The importance of the host immune system in limiting bone and joint infection is underlined by the severity and poor prognosis of bone and joint infection in patients on steroids, and those with rheumatoid disease or diabetes mellitus. The difficulty of assessing the role of the host in what is often a heterogeneous population is a major problem in clinical studies of pathogenesis and will 
only be overcome by combined molecular and clinical studies.

\section{References}

1. Waldvogel FA. Staphylococcus aureus including toxic shock syndrome. In: Mandell GL, Bennett JE, Dolin R (eds) Mandell, Douglas and Bennett's principles and practice of infectious diseases 4th edn, vol 2. New York, Churchill Livingstone. 1995: 1754-1777.

2. Smith JW, Piercy EA. Infectious arthritis. In: Mandell GL, Bennett JE, Dolin R (eds) Mandell, Douglas and Bennett's principles and practice of infectious diseases 4th edn, vol 1. New York, Churchill Livingstone. 1995: 1032-1039.

3. Mader JT, Calhoun J. Osteomyelitis. In: Mandell GL, Bennett JE, Dolin R (eds) Mandell, Douglas and Bennett's principles and practice of infectious diseases 4 th edn, vol 1. New York Churchill Livingstone. 1995: 1039-1051.

4. Cierny G, Mader JT, Pennick H. A clinical staging system of adult osteomyelitis. Contemp Orthop 1985; 10: 17-37.

5. Dubey L, Krasinski K, Hernanz-Schulman M. Osteomyelitis secondary to trauma or infected contiguous soft tissue. Pediatr Infect Dis $J$ 1988; 7: 26-34.

6. Barton LL, Dunkle LM, Habib FH. Septic arthritis in childhood. A 13-year review. Am $J$ Dis Child 1987; 141: 898-900.

7. Peltola H, Vahvanen V. A comparative study of osteomyelitis and purulent arthritis with special reference to aetiology and recovery. Infection 1984; 12: 75-79.

8. Espersen F, Frimodt-Moller N, Thandrup Rosdahl V, Skinhoj P, Bentzon MW. Changing pattern of bone and joint infections due to Staphylococcus aureus: study of cases of bacteremia in Denmark: 1959-1988. Rev Infect Dis 1991; 13: 347-385.

9. Craigen MAC, Watters J, Hackett JS. The changing epidemiology of osteomyelitis in children. J Bone Joint Surg [Br] 1992; 74: 541-545.

10. McGuire NM, Kauffman CA. Septic arthritis in the elderly $J$ Am Geriatr Soc 1985; 33: 170-174.

11. Fitzpatrick DJ, Cafferkey MT, Toner M, Beattie T, Keane CT. Osteomyelitis with methicillin-resistant Staphylococcus aureus. $J$ Hosp Infect 1986; 8: 24-30.

12. Ish-Horowicz MR, McIntyre $P$, Nade $S$. Bone and joint infections caused by multiply resistant Staphylococcus aureus in a neonatal intensive care unit. Pediatr Infect Dis $J$ 1992; 11: 82-87

13. Brause BD. Infections associated with prosthetic joints. Clin Rheumat Dis 1986; 12: 523-536.

14. Wymenga AB, van Horn JR, Theeuwes A, Muytjens HL, Slooff TJH. Perioperative factors associated with septic arthritis after arthroplasty. Prospective multicentre study of 362 knee and 2651 hip operations. Acta Orthop Scand 1992; 63: 665671.

15. Poss R, Thornhill TS, Ewald FC, Thomas WH, Batte NJ, Sledge CB. Factors influencing the incidence and outcome of infection following total joint arthroplasty. Clin Orthop 1984; 182: $117-126$

16. Petty W. The effect of methylmethacrylate on the bacterial inhibiting properties of normal human serum. Clin Orthop Rel Res 1978; 132: 266-278

17. Petty W. The effect of methylmethacrylate on bacterial phagocytosis and killing by human polymorphonuclear leukocytes. J Bone Joint Surg (Am) 1978; 60: 752-757.

18. Elek SD. Staphylococcus pyogenes and its relation to disease. Edinburgh, Livingstone. 1959: 208.

19. Lorian V. In vitro simulation of in vivo conditions: physical state of the culture medium. J Clin Microbiol 1989; 27: 24032406.

20. Zak O, Sande M. Correlation of in vitro activity of antibiotics in experimental animal models and human infection. In: Sabath LD (ed) Action of antibiotics in patients. Bern, Han Huber Publishers. 1982: 55-67.

21. Cheung AL, Fischetti VA. Variation in the expression of cell wall proteins of Staphylococcus aureus grown on solid and liquid media. Infect Immun 1988; 56: 1061-1065.

22. Clark BA, Rissing JP, Buxton TB, Best NH, Best GK. The effect of growth temperature on Staphylococcus aureus binding to type 1 collagen. Microb Pathog 1994; 17: 239-251.
23. Patti JM, Bremell T, Krajewska-Pietrasik $\mathrm{D}$ et al. The Staphylococcus aureus collagen adhesin is a virulence determinant in experimental septic arthritis. Infect Immun 1994; 62: 152-161.

24. Switalski LM, Patti JM, Butcher W, Gristina AG, Speziale P, Hook M. A collagen receptor on Staphylococcus aureus strains isolated from patients with septic arthritis mediates adhesion to cartilage. Mol Microbiol 1993; 7: 99-107.

25. Foster TJ, McDevitt D. Surface-associated proteins of Staphylococcus aureus: their possible roles in virulence. FEMS Microbiol Lett 1994; 118: 199-206.

26. Vaudaux P, Pittet D, Haeberli A et al. Fibronectin is more active than fibrin or fibrinogen in promoting Staphylococcus aureus adherence to inserted intravascular catheters. $J$ Infect Dis 1993; 167: 633-641.

27. Gemmell CG, Tree R, Patel A, O'Reilly M, Foster TJ. Susceptibility to opsonophagocytosis of protein A, alphahaemolysin and beta-toxin deficient mutants of Staphylococcus aureus isolated by allele replacement. In: Jeljaszewicz J, Cibrowski P (eds) The staphylococci. Zentralbl Bakteriol 1991; Suppl 21: 273-277.

28. Smith BG, Johnson HM. The effect of staphylococcal enterotoxins on the primary in vitro immune response. $J$ Immunol 1975; 115: 575-578

29. Kunstmann G, Schroder E, Hasbach H, Pulverer G. Immune response to toxic-shock-syndrome toxin-1 (TSST-1) and to staphylococcal enterotoxins A, B and C in Staphylococcus aureus infections. Int $J$ Med Microbiol 1989; 271: 486-492.

30. Sourek J, Výmola F, Trojanová M, Zelenková L, Matějovska V, Bergdoll MS. Enterotoxin production by Staphylococcus aureus strains isolated from cases of chronic osteomyelitis. $J$ Clin Microbiol 1979; 2: 266-268.

31. Fast DJ, Schlievert PM, Nelson RD. Nonpurulent response to Toxic Shock Syndrome Toxin 1-producing Staphylococcus aureus: relationship to toxin-stimulated production of Tumour Necrosis Factor. J Immunol 1988; 140: 949-953.

32. Abdelnour A, Bremell $T$, Holmdahl R, Tarkowski A. Clonal expansion of $\mathrm{T}$ lymphocytes causes arthritis and mortality in mice infected with toxic shock syndrome toxin-1-producing staphylococci. Eur J Immunol 1994; 24: 1161-1166.

33. Abdelnour A, Bremell T, Tarkowski A. Toxic shock syndrome toxin-1 contributes to the arthritogenicity of Staphylococcus aureus. J Infect Dis 1994; 170: 94-99.

34. Baddour LM, Lowrance C, Albus A, Lowrance JH, Anderson SK, Lee JC. Staphylococcus aureus microcapsule expression attenuates bacterial virulence in a rat model of experimental endocarditis. $J$ Infect Dis 1992; 165: 749-753.

35. Jonsson $P$, Lindberg $M$, Haraldsson I, Wadström T. Virulence of Staphylococcus aureus in a mouse mastitis model: studies of alpha hemolysin, coagulase, and protein $A$ as possible virulence determinants with protoplast fusion and gene cloning. Infect Immun 1985; 49: 765-769.

36. Bhakdi S, Tranum-Jensen J. Alpha-toxin of Staphylococcus aureus. Microbiol Rev 1991; 55: 733-751.

37. Christensson B, Fehrenbach FJ, Hedström SA. A new serological assay for Staphylococcus aureus infections: detection of IgG antibodies to $S$. aureus lipase with an EnzymeLinked Immunosorbent Assay. J Infect Dis 1985; 152: 286292.

38. Power ME, Olson ME, Domingue PAG, Costerton JW. A rat model of Staphylococcus aureus chronic osteomyelitis that provides a suitable system for studying the human infection. J Med Microbiol 1990; 33: 189-198.

39. Spagnolo N, Greco F, Rossi A, Ciolli L, Teti A, Posteraro P. Chronic staphylococcal osteomyelitis: a new experimental rat model. Infect Immun 1993; 61: 5225-5230.

40. Alderson M, Speers D, Emslie K, Nade S. Acute haematogenous osteomyelitis and septic arthritis - a single disease. J Bone Joint Surg [Br] 1986; 68: 268-274.

41. Bremell T, Lange S, Holmdahl R, Rydén C, Hansson GK, Tarkowski A. Immunopathological features of rat Staphylococcus aureus arthritis. Infect Immun 1994; 62: 2334-2344.

42. Bone RC. Gram-positive organisms and sepsis. Arch Int Med 1994; 154: 26-34.

43. Soell M, Diab M, Haan-Archipoff $\mathrm{G}$ et al. Capsular polysaccharide types 5 and 8 of Staphylococcus aureus bind specifically to human epithelial (KB) cells, endothelial cells, and monocytes and induce release of cytokines. Infect Immun 1995; 63: $1380-1386$

44. Williams RJ, Smith RL, Schurman DJ. Septic arthritis. 
Staphylococcal induction of chondrocyte proteolytic activity. Arthritis Rheum 1990; 33: 533-541.

45. Bremell T, Lange S, Yacoub A, Rydén C, Tarkowski A Experimental Staphylococcus aureus arthritis in mice. Infect Immun 1991; 59: 2615-2623.

46. Kuypers JM, Proctor RA. Reduced adherence to traumatized rat heart valves by a low-fibronectin-binding mutant of Staphylococcus aureus. Infect Immun 1989; 57: 2306-2312.

47. Arbeit RD, Karakawa WW, Vann WF, Robbins JB. Predominance of two newly described capsular polysaccharide types among clinical isolates of Staphylococcus aureus. Diagn Microbiol Infect Dis 1984; 2: 85-91.

48. Lee JC, Takeda S, Livolsi PJ, Paoletti LC. Effects of in vitro and in vivo growth conditions on expression of type 8 capsular polysaccharide by Staphylococcus aureus. Infect Immun 1993; 61: $1853-1858$.

49. Patel AH, Nowlan P, Weavers ED, Foster T. Virulence of protein A-deficient and alpha-toxin-deficient mutants of
Staphylococcus aureus isolated by allele replacement. Infect Immun 1987; 55: 3103-3110.

50. Schlievert PM. Role of superantigens in human disease. $J$ Infect Dis 1993; 167: 997-1002.

51. Mills JT, Dodel AW, Kass EH. Regulation of staphylococcal toxic shock syndrome toxin-1 and total exoprotein production by magnesium ion. Infect Immun 1986; 53: 663-670.

52. Phonimdaeng P, O'Reilly M, O'Toole PW, Foster TJ. Molecular cloning and expression of the coagulase gene of Staphylococcus aureus 8325-4. J Gen Microbiol 1988; 134: 75-83.

53. Taylor AG, Bernheimer AW. Further characterization of staphylococcal gamma-hemolysin. Infect Immun 1974; 10: 54-59.

54. Abramson C. Staphylococcal isoenzymes related to disease Zentralbl Bakteriol Microbiol Hyg 1976; Suppl 15: 1041-1054

55. Morrissy RT, Haynes DW. Acute haematogenous osteomyelitis: a model with trauma as an etiology. J Pediatr Orthop 1989; 9: 447-456. 